\title{
AMENORREAS Y LA PRUEBA DE LA PROGESTERONA-PREGNANDIOL
}

\author{
Dr. Fernando Cardona A.* \\ Dr. Jairo García G.** \\ Lic. Olga Vasseur de Pareja
}

\section{Introducción}

A la amenorrea se le describe como un síndrome (1), como un síntoma, también se le estudia (2), un síntoma que revela una patología cuando se presenta fuera de las épocas prepuberal, del embarazo, de la lactancia y de la postmenopausia:

En la actualidad el estudio metódico somático y las dosificaciones hormonales permiten hacer casi en la totalidad de las pacientes con amenorrea un diagnóstico etiológico de la misma, y su causa bien es sabido puede ser cortical, diencefálica, hipotalámica, hipofisiaria, ovárica, uterina, tiroidea, suprarrenal, carencial o psíquica.

Pueden presentarse amenorreas en donde las hormonas androgénicas juegan un papel muy importante en su etiología; en ellas se encuentran dos variaciones, la primera en donde la reacción colorimétrica del 1-3 dinitrobenzol con las hormonas denominadas genéricamente 17 cetoesteroides, revela cifras superiores a la "media normal" para la edad de la paciente; la segunda en donde estas cifras encontradas se localizan en los terrenos de la "media normal" pero en donde la proporción de las hormonas con gran actividad androgénica - co- mo la androsterona y la dehidroepiandrosterona- se encuentran muy aumentadas y disminuídas las hormonas con poca actividad androgénica, con su función cetónica también en el carzón 17 como la etiocolanolona.

En estas amenorreas en donde existe un compromiso hiperandrogénico - con cifras normales o elevadas de 17 cetoesteroides - presta gran utilidad el fraccionamiento cromatográfico de los 17 cetoesteroides para aclarar su origen suprarrenal u ovárico. Este método fue descrito por Dingemanse (3) si bien tiene un buen significado clínico, presenta el problema de su larga y delicada elaboración.

Se ha descubierto una prueba funcional denominada Prueba de la Progesterona Pregnandiol $(4,5)$ que presta ayuda para un mejor estudio de aquellas amenorreas en donde el compromiso hiperandrogénico puede estar desempeñando un importante papel etiológico, dado el carácter que tiene esta prueba dinámica de valorar la actividad androgénica de la gónada -en estos casos el ovario- estén o nó elevada la eliminación diaria de

* Profesor agregado del Departamento de Obstetricia y Ginecología Universidad de Antioquia.

** Residente del Departamento de Obstetricia y Ginecología de la Universidad de Antioquia. 
las hormonas 17 cetosteroides totales. Aún más, en los casos de amenorrea en donde existe un exceso de hormonas androgénicas que se manifiestan por los signos y por los síntomas, es posible con esta prueba de la progesterona Pregnandiol conocer si el exceso de estas hormonas tiene un origen suprarrenal u ovárico.

El presente estudio muestra los hallazgos de los autores en el terreno de las amenorreas, con esta prueba que valora la actividad androgénica gonadal.

\section{Objetivos}

1\% Tener un conocimiento de la frecuencia, en las amenorreas, de la hiperactividad androgénica.

2. En caso de amenorreas con hiperactividad androgénica conocer si su etiología es suprarrenal o gonadal - a la vez de una y otra glándula.

3. Analizar en las amenorreas si existe concordancia entre la clínica y los datos obtenidos con la Prueba de la Progesterona Pregnandiol.

4. Puntualizar con el estudio efectuado en las amenorreas la eficacia de la prueba de la Progesterona Pregnandiol, que ya había sido valorada en las poliquistosis ováricas, en la hipofunción testicular, y en los testículos feminizantes.

5: Estudiar si es de utilidad hacer la Frueba de la Progesterona Pregnandiol en las amenorreas.

\section{Material y Métodos}

Se estudiaron 28 pacientes de las 35 que en el lapso de Agosto del 72 a Agosto del 73 consultaron por amenorrea al Hospital Universitario San Vicente de Paúl.
Se hacen dosificaciones de 17 cetoesteroides totales según técnica de Dreckter (6) en total 28 dosificaciones. Se efectúan dosificaciones de pregnandiol por cromatografía en capa delgada según técnica de Waldi (7) en total 224 dosificaciones. Se hacen los exámenes que habitualmente se ejecutan para el estudio de las amenorreas.

Se estudia en todas las pacientes su comportamiento frente a la prueba progesterona - pregnandiol (8), existan o no síntomas o signos de hiperandrogenismo; se hacen en total 28 pruebas.

Esta prueba se efectúa en la forma siguiente:

a) Dosificación previa de la pregnandioluria en 24 horas.

b) Aplicación por vía intramuscular de 100 miligramos de progesterona en solución oleosa (cuando se hace la prueba en mujeres efectuarla en época posmenstrual, preferible iniciándola el día 6 del ciclo).

c) Por vía oral 0.75 miligramos de dexametasona cada ocho horas durante los siete días siguientes a la inyección de progesterona, para evitar el posible metabolismo de la progesterona hacia productos diferentes al pregnandiol, por las células de la corteza suprarrenal.

d) Dosificación de la pregnandioluria diaria hasta encontrar las cifras de la dosificación previa (en promedio 7 días para las mujeres y 4 días para los hombres).

Los resultados con esta prueba (Cuadro № 1) muestran que en los hombres normales cuando se aplica 100 miligramos de progesterona intramuscular se obtienen en total $2.0 \mathrm{mi}$ ligramos de pregnandiol en orina $y$ en 
CUADRO N: 1

RESULTADO DEL. PREGNANDIOL URINARIO PROMEDIO AL APLICAR 100 MG. DE PROGESTERONA MUSCULAR

PROGESTERONA

100 miligramos

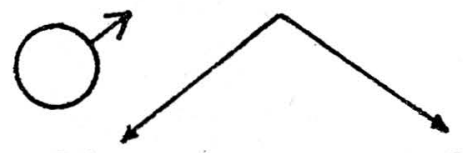<smiles>CC(C)(C)C1CCCCCCC1</smiles>

$2 . \operatorname{lmg}$

$8.0-9.0 \mathrm{mg}$

\section{PREGNANDIO L}

las mujeres normales cuando se aplica la misma dosis de 100 miligramos de progesterona intramuscular se obtienen cifras que oscilan entre 8.0 y 9.0 miligramos totales de pregnandiol en orina (4).

Cuando existe hiperactividad androgénica ovárica las cifras de pregnandiol son menores que las normales, es decir menos de 8.0 miligramos. Cuando existe insuficiencia del testículo las cifras de pregnandiol son mayores que las normales en los hombres, es decir superior a 2.0 miligramos.

\section{Resultados}

Nutrimental: (Cuadro № 2).

\section{CUADRO $N \div 2$}

NUTRIMETAL

\begin{tabular}{ccc}
\hline & 17 cetos & P. Pdiol. \\
\hline 1 & 2,9 & 8,0 \\
\hline
\end{tabular}

En un caso se encontró una amenorrea primaria con gran compromiso nutrimental, en ella clínicamente no existía una hiperactividad androgénica y sus 17 cetoesteroides estaban en los límites inferiores normales de 2.9 miligramos en diuresis de 24 horas.
La prueba de la progesterona pregnandiol reveló una cifra de eliminación total de pregnandiol de 8.0 miligramos que se pueden considerar como signo de unos ovarios con función normal de actividad androgénica.

Suprarrenal: (Cuadro N: 3 ).

\section{CUADRO NN 3}

SUPRARRENAL

\begin{tabular}{ccc}
\hline & 17 cetos & P. Pdiol. \\
\hline 1 & 16,5 & 7,7 \\
2 & 15,7 & 7,6 \\
3 & 21,0 & 8,9 \\
4 & 20,0 & 8,8 \\
\hline
\end{tabular}

En cuatro casos se encontró una eliminación de 17 cetoesteroides por encima de la "media normal", con cifras de 16.5 - 15.7 - 21.0 y 20.0 miligramos en diuresis de 24 horas. En ellas la eliminación total de pregnandiol fue de 7.7 - 7.6 - 8.9 - $8.8 \mathrm{mili}$ gramos, respectivamente.

En el primer caso no existían síntomas de hiperactividad androgénica, pero en ella la prueba de la progesterona pregnandiol dio una cifra de 7.7 miligramos que puede considerarse, en el campo aún de la hipótesis expe- 
rimental, como una razón por la cual con la sola progesterona no menstruó y sí lo logró con la combinación de estrógenos progesterona.

En la otra amenorrea que era primaria con útero rudimentario, no existían síntomas de virilización, sinembargo, tanto sus 17 cetoesteroides de 15.7 miligramos en diuresis de 24 horas y la prueba de la progesterona pregnandiol de 7.6 miligramos totales, revelan una hiperactividad androgénica moderada pero real.

Se consideran estas pacientes como dos casos de amenorrea con producción de andrógenos aumentados tanto suprarrenales como ováricos.

Los otros dos casos también considerados como suprarrenales con 17 cetoesteroides aumentados no revelan con la prueba de la progesterona pregnandiol un compromiso ovárico. En ellos sí existían síntomas francos de hiperactividad androgénica, como hirsutismo generalizado y distribución androide del vello, en uno de ellos fue necesario hacer una amputación del clítoris por encontrarse hipertrofiado.

Uterinas: (Cuadro N: 4).

\section{CUADRO NN 4}

\begin{tabular}{ccc}
\multicolumn{3}{c}{ UTERINA } \\
\hline 17 cetos & P. Pdiol. \\
\hline 1 & 6,7 & 9,1 \\
\hline
\end{tabular}

En un caso de útero rudimentario sin síntomas de hiperactividad androgénica los 17 cetoesteroides fueron normales de 6.7 miligramos en diuresis de 24 horas y la prueba de la progesterona pregnandiol dio una actividad normal de 9.1 miligramos totales.

Hipófisis: (Cuadro № 5).
CUADRO № 5

HIPOFISIS

\begin{tabular}{ccc}
\hline & 17 cetos & P. Pdiol. \\
\hline 1 & 9,0 & 5,9 \\
\hline
\end{tabular}

En una paciente con disfunción hipofisiaria postparto con galactorrea y amenarrea de 3 años de evolución, se le hicieron 3 ciclos con citrato de clomiphene y no menstruó. Dos meses más tarde se le hizo dosificación de 17 cetoesteroides que dio 9.0 miligramos en diuresis de 24 horas y la prueba progesterona pregnandiol reveló 5.9 miligramos totales. Con la sola terapia de 100 miligramos de progesterona menstruó 3 ciclos y luego entró de nuevo en amenorrea. En este caso no existían síntomas de hiperactividad androgénica, pero se piensa como hipótesis que el clomiphene estimuló la teca para dar el resultado de $5.9 \mathrm{mi}$ ligramos totales de pregnandiol.

\section{Gonadal:} 6).

a) Disgenesia gonadal: (Cuadro $N$ ?

\section{CUADRO NN 6}

GONADAL

\begin{tabular}{lcc}
\hline & $\mathbf{1 7}$ cetos & P. Pdiol. \\
\hline a) Disgenesia & & \\
gonadal & & \\
1 & 10,0 & 8,2 \\
2 & 14,8 & 6,4 \\
b) Castración & & \\
1 & 9,0 & 7,7 \\
2 & 5,0 & 7,5 \\
3 & 7,0 & 8,1 \\
\hline
\end{tabular}

Un caso de amenorrea primaria con diagnóstico de Turner típico dio 10.0 miligramos de cetoesteroides en diuresis de 24 horas y 8.2 miligramos totales en la prueba progesterona pregnandiol; no existían síntomas de virilismo. 
En otro caso se encontró a la laparotomía una cintilla fibrosa en el punto correspondiente al ovario y ausencia de útero. Los 17 cetoesteroides eran de 14.8 miligramos en diuresis de 24 horas y la prueba progesterona pregnandiol dio 6.4 miligramos totales. El cariotipo dio $X O$ y un posible $X Y$ que está en estudio. Las dosificaciones hormonales sugieren una gónada presente con hiperactividad androgénica, aunque no existan clínicamente síntomas de virilismo.

\section{b) Castración}

En dos casos se encontró amenorrea por castración quirúrgica. No existía en ellas síntomas de hiperactividad androgénica y los 17 cetoesteroides fueron de 9.0 miligramos en diuresis de 24 horas y 5.0 miligramos para el segundo caso en diuresis de 24 horas; la prueba de progesterona pregnandiol dio 7.7 miligramos totales en el primer caso y 7.5 miligramos totales en el segundo.

En un caso se hizo castración actínica por un linfoma linfocítico, no hay signos de virilización, los 17 cetoesteroides fueron de 7.0 miligramos en diuresis de 24 horas y la prueba de la progesterona pregnandiol dio 8.1 miligramos en total, normal.

c) Poliquistosis: (Cuadro N: 7).

CUADRO N: 7

GONADAL

\section{7 cetos}

P. Pdiol.

c) Poliquistosis
1
2
3

10,5

15,0

10,0

8,1

9,4

7,8

Dos de estas pacientes habían sido operadas con diagnóstico clínico de poliquistosis con hiperplasia androgénica de la teca según la prueba de la progesterona pregnandiol con cifras en la № 1 de 5.5 miligramos totales; y en la № 2 de 5.9 miligramos totales de pregnandiol.

De nuevo se encuentran en amenorrea y dieron 10.5 miligramos y 15.0 respectivamente de 17 cetoesteroides en diuresis de 24 horas y cifras con la prueba de la progesterona pregnandiol de 8.1 - 9.04 miligramos totales.

Se aprecia en ambas una función androgénica gonadal normal después de la intervención; en la segunda paciente existe un aumento de los $17 \mathrm{ce}$ toesteroides que puede explicar su hirsutismo y su obesidad.

La tercer paciente presenta fuera de su amenorrea un hirsutismo y sus 17. cetoesteroides son de 10.0 miligramos en diuresis de 24 horas y la prueba de progesterona pregnandiol es de 7.8 miligramos totales.

d) Insuficiencia ovárica: (Cuadro No. 8 ).

\section{CUADRO N 8}

GONADAL

17 cetos

P. Pdiol.

d) Insuficiencia

ovárica

\begin{tabular}{rrr}
1 & 11,0 & 9,0 \\
2 & 7,9 & 8,9 \\
3 & 8,2 & 8,2 \\
4 & 5,2 & 9,1 \\
5 & 9,1 & 8,8 \\
6 & 10,5 & 8,1 \\
\hline
\end{tabular}

Seis casos de amenorrea fueron clasificados como insuficiencia ovárica.

En ninguno de ellos había síntomas de hiperactividad androgénica y en ellos los 17 cetoesteroides fueron normales con cifras de 11.0 - 7.9 - 8.2 5.2 , 9.1 y 10.5 miligramos en diuresis de 24 horas y la prueba de la pro- 
gesterona pregnandiol reveló en su mismo orden cifras de 9.0 - 8.9 - 8.2 9.1 - 8.8 y 8.1 miligramos totales que son también normales. Es decir, la clínica y las dosificaciones hormonales mostraron concordancia.

e) Hipertecosis androgénica: (Cuadro № 9).

\section{CUADRO N: 9}

GONADAL

\begin{tabular}{lrr}
\hline & 17 cetos & P. Pdiol. \\
\hline e) Hipertricosis & & \\
androgénica & & \\
1 & 10,8 & 3,2 \\
2 & 7,6 & 6,1 \\
3 & 13,6 & 6,1 \\
4 & 5,2 & 6,5 \\
5 & 9,0 & 7,8 \\
6 & 11,3 & 7,6 \\
\hline
\end{tabular}

En 4 pacientes con amenorera existía una franca hiperactividad androgénica, con distribución morfológica androide, hirsutismo y obesidad. En ellas los 17 cetoesteroides eran normales con cifras de 10.8 - 7.6 - 13.6 y 5.2 miligramos en diuresis de 24 horas, pero la prueba de la progesterona pregnandiol reveló cifras muy bajas, es decir ovarios con gran actividad andragénica con resultados de 3.2 6.1 - 6.1 y 6.5 miligramos totales respectivamente.

En otras dos pacientes el cuadro de hiperactividad androgénica era menos acentuado y tenía 17 cetoesteroides normales de 9.0 y 11.3 miligramos en diuresis de 24 horas y una prueba de la progesterona pregnandiol con cifras de 7.8 y 7.6 miligramos totales, respectivamente.

f) Orotestis: (Cuadro No 10).

En un caso de la consulta externa de endocrinología pediátrica se encontró un falo pequeño, hipospadia y escroto sin contenido, se hizo laparoto-

\section{CUADRO N: 10}

GONADAL

17 cetos

P. Pdiol.

f) Ovotestis

1

16,1

3,2

mía y se extirpó unilateralmente el tejido gonadal que fue informado como ovotestis. El paciente abandonó el hospital y regresa ahora a los 7 años de evolución con una "Ginecomastia" bilateral. Los 17 cetoesteroides son actualmente de 16.1 miligramos en diuresis de 24 horas y la prueba de la progesterona pregnandiol reveló 3.2 miligramos totales. Se exploró de nuevo y se extrajo una formación que fue informada como cuerpo uterino. En este caso la clínica por el crecimiento del tejido mamario y la bioquímica por el resultado de la prueba de la progesterona pregnandiol revelan la existencia de una gónada activa.

\section{Comentarios}

Los resultados obtenidos permiten valorar el primer objetivo propuesto acerca de si es frecuente o nó la hiperactividad androgénica en las amenorreas. En efecto de las 28 amenorreas estudiadas se encontró en $13 \mathrm{ca}$ sos, que ella existía.

El segundo objetivo pudo revelar que en dos casos existía una hiperactividad androgénica tanto suprarrenal como ovárica. En dos casos suprarrenal puros y en 9 casos gonadales puros.

El tercer objetivo mostró concordancia entre la hiperactividad androgénica clínica y la prueba de la Progesterona Pregnandiol en 11 de los 13 casos. Los dos casos que indicaron discordancia fueron el uno por causa iatrogénica con citrato de clomiphene 
y el otro por un posible -aún no confirmado - testículo feminizante.

El cuarto objetivo se logró al mostrar una vez más la eficacia de la Prueba de la Progesterona Pregnandiol como índice que revela la actividad androgénica de la gónada, cuyos resultados fueron lógicos en la totalidad de las 28 pacientes estudiadas.

El quinto objetivo indica que el clínico obtiene un más claro conocimiento endocrinológico de su paciente si efectúa esta prueba, y que por lo tanto la Prueba de la Progesterona Pregnandiol es útil y eficaz en el estudio de las amenorreas.

\section{Resumen}

Se estudian 28 pacientes con amenorrea en las cuales mediante dosificación de los 17 cetoesteroides y la Prueba de la Progesterona Pregnandiol se analiza la existencia 0 no en ellas de hiperactividad androgénica, y su origen ovárico o suprarrenal. Se muestra la importancia de la Prueba de la Progesterona Pregnandiol en el estudio de las amenorreas.

\section{Summary}

A study is made of 28 patients with amenorrhea which, by means of a dose of 17 cetoesteroids and the test of Pregnandio progesterone, are analyzed for presence of an androgenical hyper-activity and their ovary or suprarenal origin. The importance of the Pregnandio progesterone test is emphasized for the study of amenorrhea.

\section{BIBLIOGRAFIA}

1 CONILL V. Tratado de Ginecología. P. 102. Ed. Labor, Madrid, 1956.

2 CARDONA F., ARISTIZABAL H.: Progesterona y Pregnandiol. Rev. Col. Obst. y Gin. XVIII, 137-156, 1967.

3 CARDONA P. N. y col. Poliquistosis ovárica. Rev. Col. de Obst. y Gin. 19: 171-197, 1968.

4 CARDONA F. La prueba de la ProgesteronaPregnandiol en el testículo feminizante. Rev. Col. Sec. Collem. Endocrinología. VIII: 2, 21-24, 1972.

5 DINGEMANSE E., y col.: Clinical method for chromatografic colorimetric determination of urinary 17 Ketosteroids: normal adults. J. Clin. Endocr. Metab. 12: 66, 1952.

6 DREKTER I. I. y col. Determination of urinary steroids preparation of pigment-free extracts and simplified procedure for estimation of total 17 ketosteroids. J. Clin. Endocrinol. 12: 55, 1952.

7 GUILLON J.; HOULNE P. Enciclopedia medica Quirurgicale, Ginecología. 156 A10, 1968.

8 WALDI D. Einfeche quantitative Schenellbastimmung von Pregnandiol auf Dunnschicht platten zum Frukschwanger schafisnach weis und zur Uber wachung des Cyclus bei der Frau, Klin. Wschr. 40: 827.830, 1962. 